\title{
An elliptic property of parabolic trajectories
}

\author{
J. L. Fernández-Chapou, A. L. Salas-Brito, C. A. Vargas \\ Departamento de Ciencias Básicas, \\ Universidad Autónoma Metropolitana-Azcapotzalco, \\ Apartado Postal 21-267, Coyoacán 04000 D. F., Méxicd*
}

\begin{abstract}
The curve joining the points of maximum height in the parabolas of ideal projectile motion is shown to be an ellipse. Some features of the motion are illustrated with the help of such ellipse.
\end{abstract}


In the last years several contributions have analysed the old but ever interesting problem of the properties of projectile motion. $\frac{1.2 .3 .4 .5 .6}{1 n}$ this note we want to pinpoint that an ellipse is also related to the parabolas traced by projectiles launched in a constant field. The ellipse appears as the curve connecting the points of maximum height in the parabolas.

To obtain this little known fact, recall that the coordinates of projectiles launched from the origin in the $x-y$ plane with the same initial speed $v_{0}$ but different launching angles are

$$
x=v_{0} t \cos \alpha, \quad y=v_{0} t \sin \alpha-\frac{1}{2} g t^{2},
$$

where $\alpha$ is the launching angle. From these equations it is simple to obtain the time for reaching the maximum height as $t_{m}=v_{0} \sin \alpha / g$. Therefore, the coordinates of the projectile at $t_{m}$ are

$$
x_{m}=\frac{v_{0}^{2}}{g} \cos \alpha \sin \alpha, \quad y_{m}=\frac{1}{2} \frac{v_{0}^{2}}{g} \sin ^{2} \alpha,
$$

where $y_{m}$ is the maximum height attained and $x_{m}$ the corresponding horizontal coordinate. It should be noted that the trajectories described by Eq. (II) are symmetric respect to the point $\left(x_{m}, y_{m}\right)$ and so the range of a projectile launched at an angle $\alpha$ is $R=2 x_{m}=$ $2 \cos \alpha \sin \alpha v_{0}^{2} / g$. With the help of the trigonometric identities $2 \cos \alpha \sin \alpha=\sin 2 \alpha$ and $2 \sin ^{2} \alpha=1-\cos 2 \alpha$, Eqs. (2) can be casted in the form

$$
x_{m}=\frac{v_{0}^{2}}{2 g} \sin 2 \alpha, \quad y_{m}=\frac{v_{0}^{2}}{4 g}(1-\cos 2 \alpha) .
$$

Eliminating the angle $\alpha$ from (3) we get the loci of points of maximum height

$$
\frac{x_{m}^{2}}{a^{2}}+\frac{\left(y_{m}-b\right)^{2}}{b^{2}}=1,
$$

where we have defined $a=v_{0}^{2} / 2 g$, and $b=v_{0}^{2} / 4 g, i . e . \quad a=2 b$. Eq. (44) represents an ellipse centered at point $(0, b)$ with minor and major axes given, respectively, by $2 b$ and $2 a$. The eccentricity is $e=\sqrt{3} / 2$ and does not depend on any details of the motion. See Fig. 1 for a graph of the ellipse and of some of the projectile trajectories that generate it.

Discussing different ways of obtaining a result may enhance student retention and understanding. To obtain (4) in another way, we can start from the gedanken experiment of simultaneously firing from the origin many projectiles with the same initial speed $v_{0}-e . g$. the case of an exploding firecracker $\underline{\underline{6}}$ At any later time, $t$, the projectiles are on the curve 


$$
x^{2}+\left(y+\frac{1}{2} g t^{2}\right)^{2}=v_{0}^{2} t^{2} .
$$

That is, the projectiles are located on an expanding and freely falling circle. To see it, take the point of view of a free-falling frame where gravity vanishes ${ }^{7}$. In this frame the projectiles are on a expanding circle of radius $v_{0} t$ - as can be derived from Eqs. (11) with $g=0$. But in the frame where gravity is acting, the center of mass is falling at a constant acceleration $g \neq 0$, hence it is located at $\left(0,-g t^{2} / 2\right)$ and Eq. (5) follows. When we substitute the time $t_{m}$ in this equation we obtain that the points of maximum height, $\left(x_{m}, y_{m}\right)$, satisfy

$$
x_{m}^{2}+\left(y_{m}+\frac{1}{2} \frac{v_{0}^{2}}{g} \sin ^{2} \alpha\right)^{2}=\frac{v_{0}^{4}}{g^{2}} \sin ^{2} \alpha .
$$

To eliminate $\sin ^{2} \alpha$ from (6) we employ Eq. (3) and obtain Eq. (44) again. In fact, we can do an identical calculation making not reference whatsoever to particles on a circle, since the equation of the path of a single projectile in the falling frame is $x^{\prime 2}+y^{\prime 2}=v_{0}^{2} t^{2}$ - the primed variables being coordinates in such frame.

Our ellipse also affords yet another solution to the problem of maximizing the range in projectile motion since the right vertex of it (see Fig. 1) corresponds precisely to such a case.

Can the ellipse (4) be characterized in any other way? It can be shown to be also the locus of points where the radial $(\dot{r})$ component of the velocity vanishes. Stablishing this property can be a simple exercise in intermediate mechanics. It can also be proved that as long as the downward branch $(i$. e. the one in which $\dot{y}<0)$ of a projectile path is within the ellipse then the projectile is necessarily approaching the origin otherwise it is getting away from it.

\section{Acknowledgments}

ALSB has been partially supported by a PAPIIT-UNAM grant 108302. Helpful comments from D Crida, H Kranken, and K Bielii are gratefully acknowledged. Gabriela Báez is acknowledged for spotting a gross misprint in the typescript. This work is for Mileva Sofía.

* Electronic address: jlfc@correo.azc.uam.mx,asb@correo.azc.uam.mx,cvargas@correo.azc.uam.mx 
1 D. Bajc "Maximum range in ideal projectile motion-A generalization," Am. J. Phys. 58 408-409 (1990).

2 S. K. Bose "Thoughts on projectile motion," 53 175-176 (1985).

3 A. P. French "The envelopes of some families of projectile trajectories," Am. J. Phys. 61, 805-811 (1993).

4 R. H. Price and J. D. Romano "Aim high and go far - Optimal projectile lauch at angles greater than 45," Am. J. Phys. 66 109-113 (1998).

5 M. Baće, S. Ilijić, Z. Narančić, and L. Bistričić "The envelope of projectile trajectories," Eur. J. Phys. 23 637-642 (2002).

6 E. I. Butikov "Comment on 'The envelope of projectile trajectories'," Eur. J. Phys. 24 L5-L9 (2003).

7 E. F. Taylor and J. A. Wheeler Spacetime physics (Freeman, New York, 1992) Ch. 2. 
FIG. 1: The ellipse of maximum heights associated with a family of parabolic trajectories launched with the same initial speed (thick black curve). The center of the ellipse is at $(0, b)$ and its eccentricity is $\sqrt{3} / 2$. The ten lighter curves are some of the projectile paths giving birth to it. The gray curve is the parabolic envelope of the family of projectile trajectories. The numbers shown on the right of the $y$-axis correspond to the launching angle of the nearest parabola. The symmetric parabolas on the left correspond to projectiles launched with the same speed but complementary angles. This plot makes apparent that the maximum range is reached for $\alpha=45^{\circ}, i$. e. it corresponds to the right vertex of the ellipse, and is $R_{m}=2 a$. 


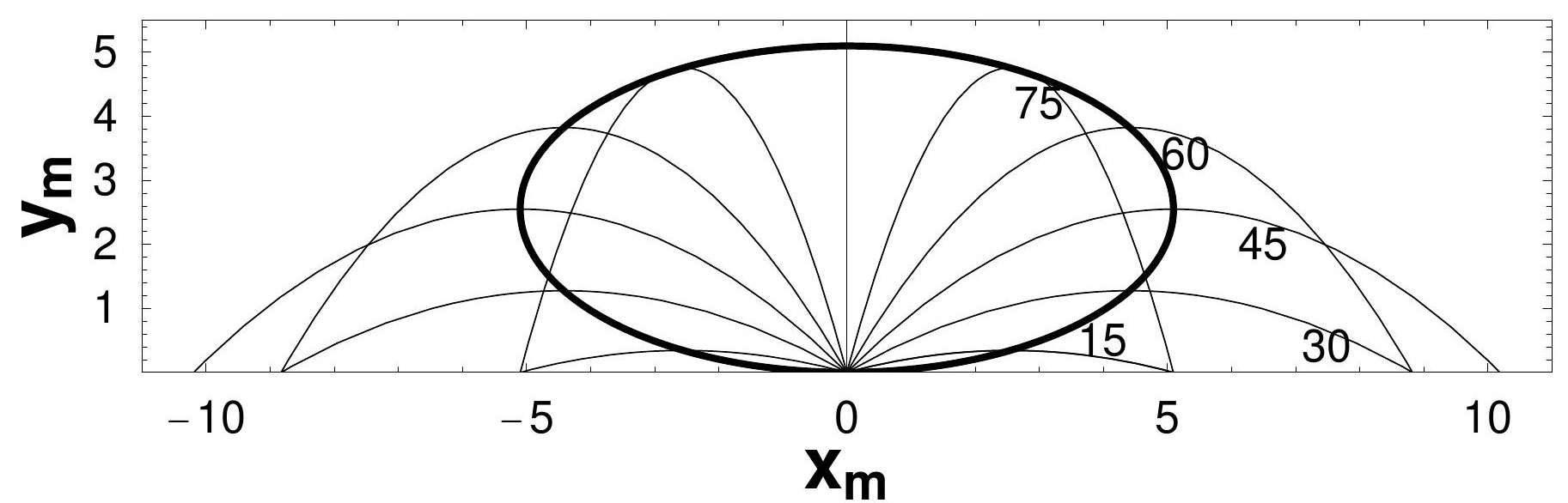

